\section{Therapeutic management of a symptomatic Kaposi's sarcoma patient with renal failure undergoing haemodialysis: A case report}

\author{
Anna Passarelli, ${ }^{1,2}$ Giovanna Galdo, ${ }^{3}$ \\ Teresa Pellegrino, ${ }^{4}$ Giandomenico \\ Roviello, ${ }^{5}$ Michele Aieta, ${ }^{1}$ \\ Gabriella Aviello, ${ }^{6}$ Raffaele Conca ${ }^{1}$ \\ ${ }^{1}$ Unit of Medical Oncology, Department \\ of Onco-Hematology, Centro di \\ Riferimento Oncologico della Basilicata \\ (IRCCS-CROB), Rionero in Vulture \\ (PZ); ${ }^{2}$ Department of Urology and \\ Gynecology, Istituto Nazionale Tumori \\ IRCCS Fondazione G. Pascale, Naples; \\ ${ }^{3}$ Oncologic Dermatology Unit, \\ Department of Onco-Hematology, \\ IRCCS-CROB, Centro di Riferimento \\ Oncologico della Basilicata (IRCCS- \\ CROB), Rionero in Vulture (PZ); \\ ${ }^{4}$ Nuclear Medicine Department, Centro \\ di Riferimento Oncologico della \\ Basilicata (IRCCS-CROB), Rionero in \\ Vulture (PZ); ${ }^{5}$ Department of Health \\ Sciences, University of Florence; \\ ${ }^{6}$ Department of Pharmacy, School of \\ Medicine and Surgery, University of \\ Naples Federico II, Naples, Italy
}

\begin{abstract}
Kaposi's sarcoma (KS) is a rare inflammation-based vascular cancer involving the skin. The viral aetiology of $\mathrm{KS}$ is the Human Herpesvirus 8. KS may be frequently diagnosed in immunosuppressed kidneytransplanted patients, while is less common in patients with dialysis. It is known that various immunological abnormalities can lead to impaired immune status in uremic patients. It is noteworthy that despite the incidence of $\mathrm{KS}$ in patients with renal impairment, only few cases have reported efficacy and safety profile of KS targeting anti-cancer drugs in this kidney disease population. Herein, we report the first case of a symptomatic KS patient with renal disease in haemodialysis and focus on its therapeutic management. We also review the main data available from literature regarding the safety of KS therapy in dialysis patients.
\end{abstract}

\section{Introduction}

Kaposi's sarcoma (KS) is a rare inflam- matory proliferative tumor originating from vascular endothelium and caused by Kaposi's sarcoma-associated herpesvirus (KSHV), also known as human herpesvirus 8 (HHV-8). ${ }^{1} \mathrm{HHV}-8$ is found in most KS lesions where establishes a latent infection in target cells, including B lymphocytes and endothelial cells. KS is characterized by increased proliferation of HHV-8-infected spindle cells and abnormal neovasculature formation. This type of cancer primarily involves the skin, however oral mucosa, lymph nodes, and viscera may also be affected.

Typically, KS occurs in immunosuppressed patients, particularly those receiving organ (e.g. kidney) transplant and patients with acquired immunodeficiency syndrome (AIDS). ${ }^{2}$ Nevertheless, KS has been described also in non-immunosuppressed patients, with several data reports showing increased KS incidence in patients undergoing dialysis treatment. Indeed, during prolonged haemodialysis (HD) treatment, the carcinogenesis process may be triggered by either cell-mediated or humoral impairment of the immune system, resulting in a greater susceptibility to oncogenic viral infections.

Several studies have shown a high genomic damage in patients undergoing dialysis. This cancer-triggering event seems due to specific mechanisms of genotoxic damage including dysfunctional DNA repair, reduced antioxidant activity (typical of chronic renal failure), release of uremic toxins during dialysis procedures, and increased production of inflammatory cytokines. $^{3-5} \quad$ Moreover, significant improvements made in the management of patients with end-stage renal disease (ESRD) have led to prolonged survival, which may contribute to increased incidence risk of KS malignancy. ${ }^{6}$

The therapeutic strategy to treat KS depends on several factors, i.e. extension of disease, number of skin lesions, rate of tumor growth, symptoms and comorbidities. $^{7-9}$ Therefore, KS management may range from local therapy of cutaneous disease to systemic therapy of visceral and symptomatic lesions. ${ }^{10}$ Cryotherapy, ionizing radiation, surgical excision, and photodynamic therapy have been widely used to treat localized skin lesions. ${ }^{11,12}$ Conversely, there are some aggressive KS variants with early involvement of skin, lymph nodes and visceral organs, for which chemotherapy is needed. Anti-cancer drugs, single or in combination, have shown an overall response rate up to $80 \%$. For patients with comorbidities (including renal impairment treated with HD) a minimally invasive treatment or personalized chemotherapy
Correspondence: Anna Passarelli, Department of Urology and Gynecology, IRCCS Fondazione Pascale, Naples, Italy.

E-mail: passarellian@libero.it

Key words: Kaposi sarcoma; Human herpesvirus 8; Chronic renal impairment; Haemodialysis; Pomalidomide.

Conflict of interest: The authors declare no potential conflict of interest.

Funding: None.

Ethical approval and consent to participate: Written informed consent was obtained from the patient.

Availability of data and material: Data and materials are available by the authors.

Please cite this article as: Passarelli A, Galdo $G$, Pellegrino $T$, et al. Therapeutic management of a symptomatic Kaposi's sarcoma patient with renal failure undergoing haemodialysis: A case report. Dermatol Rep 2022;14:9113.

Received for publication: 25 February 2021. Accepted for publication: 21 June 2021

This work is licensed under a Creative Commons Attribution-NonCommercial 4.0 International License (CC BY-NC 4.0).

${ }^{\circ}$ Copyright: the Author(s), 2022

Licensee PAGEPress, Italy

Dermatology Reports 2022; 14:9113

doi:10.4081/dr.2022.9113

dosage adjustment may be required. ${ }^{13}$

Unfortunately, only a few clinical data regarding both safety and efficacy of the anti-cancer drugs in KS patient with disrupted renal function are currently available. More importantly, there are no defined guidelines regarding the administration of the most cytotoxic drugs employed in the treatment of cancer to patients undergoing HD.

Herein, we report a complex therapeutic management of a symptomatic KS patient with chronic renal failure undergoing HD.

\section{Case Report}

In 2016, a 66-year-old white man presented with a six-month history of 2 growing asymptomatic red-purple nodules located on his left leg and right temporal region. Radical excisional biopsies of the two isolated nodules were consistent with $\mathrm{KS}$, associated with positive immunohistochemical staining for HHV-8. HIV serology was 
negative. The patient had a personal history of end-stage renal disease for over ten years, obesity, type II diabetes and hypertensive heart disease in pharmacological therapy. Creatinine value was $7.0 \mathrm{mg} / \mathrm{dL}$; therefore the patient was dialysis-dependent [stage 5 chronic kidney disease (CKD)], with a calculated estimated glomerular filtration rate (eGFR) of $17 \mathrm{~mL} / \mathrm{min} / 1.73 \mathrm{~m}^{2}$. In spite of these outcomes, the patient was functioning well, as indicated by an Eastern Cooperative Oncology Group performance status of 1 .

Two years after the histological diagnosis, erythematous and violaceous papules, nodules, and plaques with lichenification appeared bilaterally on legs and feet (Figure 1a). In order to complete the clinical stage assessment, the patient underwent wholebody ${ }^{18}$ FDG-PET scan. Imaging analyses showed focal uptake in the right pre-auricular region, in several lymph nodes located in the left lateral cervical area and iliac and inguinal region bilaterally, in anterior region of the left gluteus muscle and quadriceps ipsilateral as well as in proximity of gastrocnemius bilaterally. Furthermore, an imbibition of the subcutaneous tissues of the legs bilaterally appeared mostly on the right such as a lymphangiosis (Figure 1b).

Following a multidisciplinary discus- sion and cardiological evaluation, the patient started systemic chemotherapy with three cycles of pegylated liposomal doxorubicin (PLD) at standard dose, thus obtaining a clinical benefit (Figure 1c). The PLD treatment was prolonged for further six cycles, obtaining a clinical benefit and a partial metabolic response (Figure 1d).

Four months after stopping chemotherapy, the patient showed an objective and radiological disease progression characterized by a high metabolic activity to the iliac and inguinal lymph nodes bilaterally, right popliteal fossa, both legs and medial area of the left foot (Figure 1e). Patient was then treated weekly with paclitaxel chemotherapy at standard dose for a total of six cycles. Notably, the infusion of chemotherapy was always followed by HD treatment. The chemotherapy treatment was well tolerated. Indeed, no treatment-related toxicities were observed. The patient achieved a clinical benefit and a complete metabolic response (Figure 1f).

After six months of exclusive clinical observation, the patient showed a metabolic progression of disease at the lymph nodes located in retrocaval, iliac bifurcation, bilateral common iliac, bilateral external iliac, superficial and deep bilateral inguinal regions (Figure $2 \mathrm{a}$ ). In addition, the patient showed a clinical progression characterized by symptomatic hyperpigmented patches, ulcerated plaques involving both lower limbs associated with a conspicuous edema (Figure 2a). At this point, treatment with gemcitabine was started at $1000 \mathrm{mg} / \mathrm{m}^{2}$ on days 1 and 8 every four weeks, leading to a rapid reduction of the growing lesions and a stabilization of several skin nodules (Figure 2b). The ${ }^{18}$ FDG-PET scan confirmed this clinical improvement after three months of chemotherapy, showing partial metabolic response on lymph nodes and excluding a visceral progression (Figure $2 \mathrm{~b}$ ).

According with the nephrologist consultant, the patient underwent HD the day after gemcitabine administration. The toxicity was mild and standard for gemcitabine, with no grade 3-4 adverse events. The gemcitabine treatment allowed a clinical disease control for approximately six months. Subsequently, the patient developed signs of disease progression mainly in the lower limbs bilaterally where severe edema, swelling, erythema of the feet appeared (Figure 2c). This disease progression provoked severe functional impotence with limited walking. Oral therapy with etoposide with a dose reduction of $50-60 \%$ was started, regardless dialysis timing.

In July 2020, a new progression on the
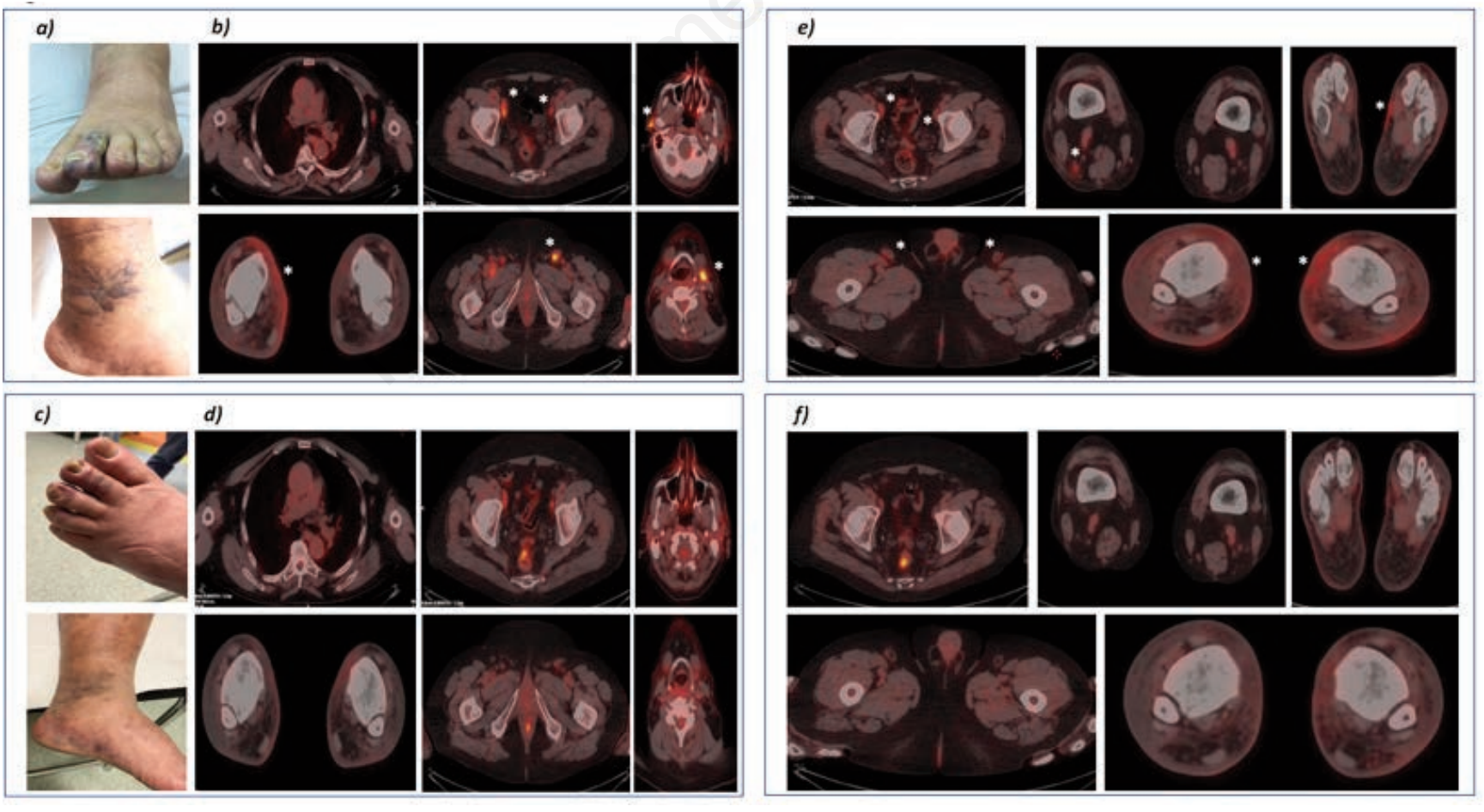

Figure 1. Basal photo assessment before PLD therapy (a); 18FDG-PET scan before PLD therapy (b); photo assessment after PLD therapy showing clinical benefit (c); 18FDG-PET scan showing partial metabolic response to PLD therapy (d). 18FDG-PET scan showing metabolic progression disease before starting paclitaxel (e); radiological evaluation showing a complete metabolic response to paclitaxel therapy (f). 
inferior limbs associated with metabolic progression occurred (Figure 2d). Thus, the patient received a fifth-line therapy with pomalidomide orally for 21 days of a 28 cycle. Pomalidomide was administered post-dialysis on the days of dialysis

In August 2020 the patient died due to the onset of acute renal failure. This occurred 4 weeks after starting pomalidomide treatment. He survived for 24 months from the beginning of the systemic treatment.

\section{Discussion}

Increased cancer risk in patients with ESRD occurs after kidney transplantation and during dialysis procedures. To this regard, there have been several reports of various immunological abnormalities leading to impaired immune status in uremic patients. These factors might have contributed at least in part to the pathogenesis of KS in our patient. Anyway, we believe that further studies regarding the relationship between dialysis and the incidence of KS are needed.

Regarding the use of antineoplastic agents for the treatment of $\mathrm{KS}$ in patients undergoing $\mathrm{HD}$, the literature is limited to a few case reports or small case series. The absence of knowledge about the anticancer drug management in this patient population can contribute to an inappropriate and dangerous use of chemotherapy, thus increasing the risk of suboptimal treatment or worsening chemotherapy toxicity profile. However, there is some evidence supporting the optimal mode of administration for some drugs that are more widely used in clinical practice.

Interestingly, the therapeutic management of our patient was always shared in a multidisciplinary context and in agreement with the nephrologist consultant in order to establish both the pharmacokinetic profile of the drugs used and the correct timing of the dialysis procedure.

Since PLD is considered the treatment of choice for patients with KS, ${ }^{14}$ our patient received this treatment. Moreover, it has been reported in the literature that the use of anthracyclines such as PLD is allowed at standard dose even in patients in HD, without additional toxicity observed. ${ }^{13}$ Indeed, anthracyclines and their major metabolites are poorly metabolized by the kidney, thus dose adjustment may not be necessary, and the administration should be done after dialysis. As reported in our case report, the chemotherapy was well tolerated without causing a worsening of renal function.
In consideration of its antiangiogenetic activity, paclitaxel is considered the best second line choice after PLD or the first choice in selected KS patients. ${ }^{15,16}$ For this reason, our patient received paclitaxel. Importantly, the renal contribution to the clearance of paclitaxel is negligible. In addition, a few reports confirm that paclitaxel pharmacokinetic profile does not differ significantly in patients with ESRD compared to those with normal kidney function. The efficacy and tolerance data of paclitaxel are satisfactory, thus the dosage adjustments and the timing of dialysis is unimportant.

Due to the further progression of disease, our patient received a third line choice, the nucleoside analogue gemcitabine. Renal metabolism of gemcitabine and its metabolite contributes for $10 \%$ and $90 \%$ respectively, to their complete removal. Although the gemcitabine toxicity observed in patients undergoing HD is generally comparable to that reported in patients with preserved renal function, it has been reported that to reduce the incidence of side effects, HD should be started within 6-12 hours after chemotherapy administration. This protocol was applied in our case.

Etoposide is mainly metabolized by the kidneys. Indeed, several studies have reported a rise in the AUC and a prolonged

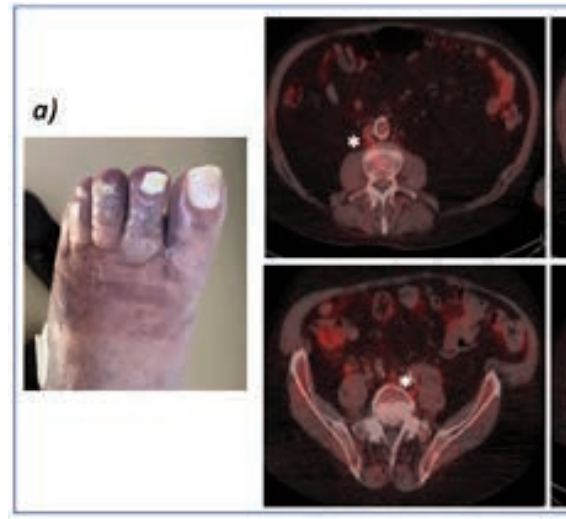

c)
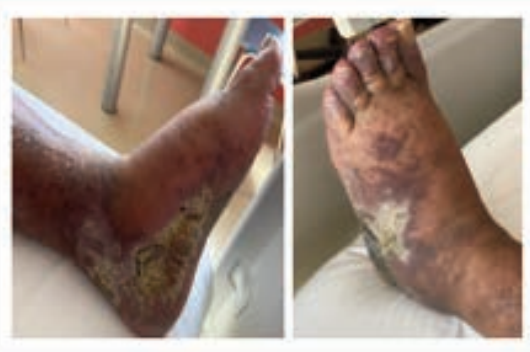
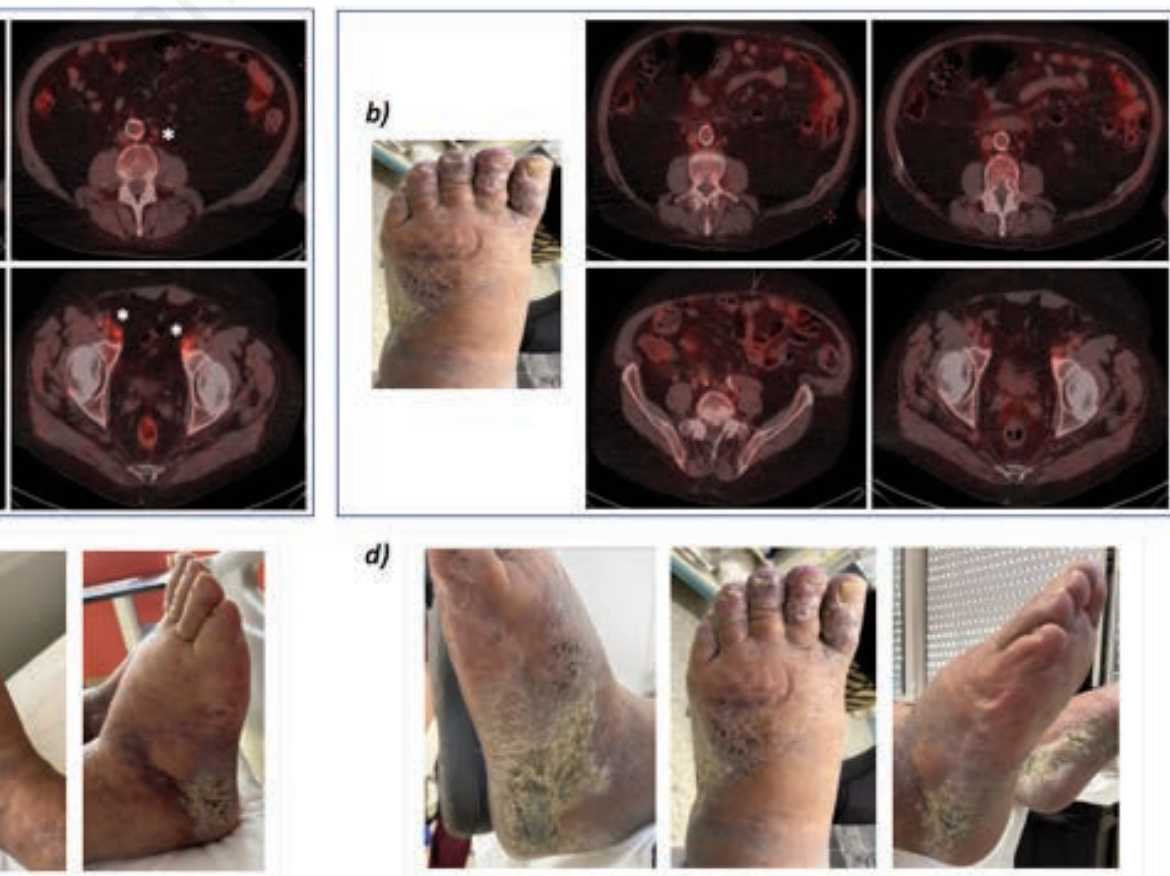

d)
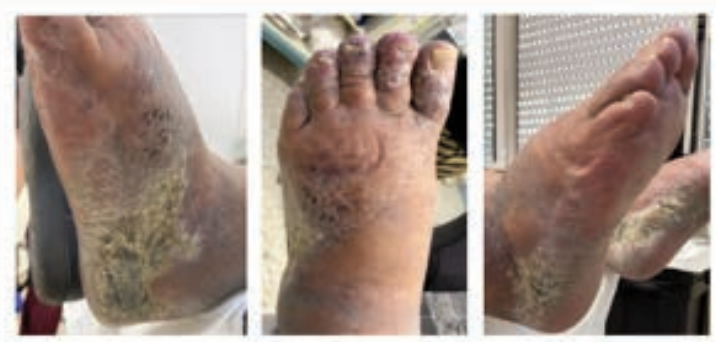

Figure 2. Photo assessment and radiological evaluation showing clinical and metabolic progression before gemcitabine (a) and the benefit achieved by gemcitabine treatment (b). Photo assessment of clinical progression of disease before etoposide treatment (c) and before oral pomalidomide therapy $(\mathbf{d})$. 
half-life of etoposide, suggesting that a dose reduction is essential in order to avoid haematological toxicity. On the basis of these data, a dose reduction of $50 \%$ should be recommended in patients undergoing dialysis. ${ }^{17,18}$ Interestingly, etoposide is not removed by $\mathrm{HD}$, thus it can be taken regardless of dialysis session.

Finally, pomalidomide was administered to our patient in further clinical and radiological progression. This choice was made based on its promising efficacy data in adult KS patients (with or without HIV infection) in terms of overall response rates $(\sim 71 \%)$ and median duration of response ( $\sim 10.5$ months).${ }^{19}$ Pomalidomide is an ana$\log$ of thalidomide with anti-angiogenic, immunomodulatory, and direct anti-neoplastic activities. It is extensively metabolized by the liver, with approximately $2 \%$ of the drug being eliminated in the urine, thus it does not require dose adjustments for patients with renal impairment. ${ }^{20}$ Despite renal dysfunction has no influence on both pomalidomide clearance and plasma concentration, the dialysis appears to increase its clearance thus removing the drug from blood circulation. In specific, pomalidomide exposure increased approximately $35 \%$ in patients with severe renal failure requiring dialysis on non-dialysis days. Although it cannot be excluded that pomalidomide therapy caused worsening of renal function and death in our patient, it should always be recommended that pomalidomide therapy is administered after HD.

\section{Conclusions}

In conclusion, this is the first clinical case to describe the complexity of managing symptomatic KS patient with renal failure and undergoing HD. Our case reports and supports the tolerance profile of multiple drug strategies in this complex patient population when a multidisciplinary team is consulted. Undoubtedly, further studies are needed to improve the clinical management of this specific population of patients.

\section{References}

1. Chang Y, Cesarman E, Pessin MS, et al. Identification of herpesvirus-like DNA sequences in AIDS-associated Kaposi's sarcoma. Science 1994;266:1865-9.

2. Yarchoan R, Uldrick TS. HIVAssociated Cancers and Related Diseases. N Engl J Med 2018;378: 1029-41.

3. Descamps-Latscha B, Jungers P, WitkoSarsat V. Immune system dysregulation in uremia: role of oxidative stress. Blood Purif 2002;20:481-4.

4. Birkeland SA. Uremia as a state of immune deficiency. Scand J Immunol 1976;5:107-15.

5. Kato S, Chmielewski M, Honda H, et al. Aspects of immune dysfunction in end-stage renal disease. Clin J Am Soc Nephrol 2008;3:1526-13.

6. Maisonneuve P, Agodoa L, Gellert R, et al. Cancer in patients on dialysis for end-stage renal disease: an international collaborative study. Lancet 1999;354: 93-9.

7. Franceschi S, Maso LD, Rickenbach M, et al. Kaposi sarcoma incidence in the Swiss HIV Cohort Study before and after highly active antiretroviral therapy. Br J Cancer 2008;99:800-4.

8. Grabar S, Abraham B, Mahamat A, et al. Differential impact of combination antiretroviral therapy in preventing Kaposi's sarcoma with and without visceral involvement. J Clin Oncol 2006;24: 3408-14.

9. Gallafent JH, Buskin SE, De Turk PB, et al. Profile of patients with Kaposi's sarcoma in the era of highly active antiretroviral therapy. J Clin Oncol 2005;23:1253-60.

10. Epstein JB. Treatment of oral Kaposi sarcoma with intralesional vinblastine. Cancer 1993;71:1722-5.

11. Bodsworth NJ, Bloch M, Bower M, et al. International Panretin Gel KS Study Group. Phase III vehicle-controlled, multi-centered study of topical alitretinoin gel $0.1 \%$ in cutaneous AIDS-related Kaposi's sarcoma. Am J Clin Dermatol 2001;2:77-87.

12. Donato V, Guarnaccia R, Dognini J, et al. Radiation therapy in the treatment of HIV-related Kaposi's sarcoma. Anticancer Res 2013;33:2153-7.

13. Predazzoli P, Silvestris N, Santoro A, et al. Management of patients with endstage renal disease undergoing chemotherapy: recommendations of the Associazione Italiana di Oncologia Medica (AIOM) and the Società Italiana di Nefrologia (SIN). ESMO Open 2017;19:e000167.

14. Northfelt DW, Dezube BJ, Thommes JA, et al. Pegylated-liposomal doxorubicin versus doxorubicin, bleomycin, and vincristine in the treatment of AIDS-related Kaposi's sarcoma: results of a randomized phase III clinical trial. J Clin Oncol 1998;16:2445-51.

15. Tulpule A, Groopman J, Saville MW, et al. Multicenter trial of low-dose paclitaxel in patients with advanced AIDSrelated Kaposi sarcoma. Cancer 2002;95:147-54.

16. Gill PS, Tulpule A, Espina BM, et al. Paclitaxel is safe and effective in the treatment of advanced AIDS-related Kaposi's sarcoma. J Clin Oncol 1999;17:1876-83.

17. Evans SR, Krown SE, Testa MA, et al. Phase II evaluation of low-dose oral etoposide for the treatment of relapsed or progressive AIDS-related Kaposi's sarcoma: an AIDS Clinical Trials Group clinical study. J Clin Oncol 2002;20:3236-41.

18. Sprinz E, Caldas AP, Mans DR, et al. Fractionated doses of oral etoposide in the treatment of patients with aids-related kaposi sarcoma: a clinical and pharmacologic study to improve therapeutic index. Am J Clin Oncol 2001;24:17784.

19. Polizzotto MN, Uldrick TS, Wyvill $\mathrm{KM}$, et al. Pomalidomide for Symptomatic Kaposi's Sarcoma in People With and Without HIV Infection: A Phase I/II Study. J Clin Oncol 2016;34:4125-31.

20. Li Y, Wang X, O'Mara E, et al. Population pharmacokinetics of pomalidomide in patients with relapsed or refractory multiple myeloma with various degrees of impaired renal function. Clin Pharmacol 2017;9:133-45. 